\title{
BMJ Open Smartphone-Enhanced Low-Threshold Intervention for adolescents with Anorexia Nervosa (SELTIAN) waiting for outpatient psychotherapy: study protocol of a randomised controlled trial
}

\author{
David R Kolar, Florian Hammerle, Ekkehart Jenetzky, Michael Huss
}

To cite: Kolar DR, Hammerle F, Jenetzky E, et al. SmartphoneEnhanced Low-Threshold Intervention for adolescents with Anorexia Nervosa (SELTIAN) waiting for outpatient psychotherapy: study protocol of a randomised controlled trial. BMJ Open 2017;7:e018049. doi:10.1136/ bmjopen-2017-018049

- SUPPLEMENT ONE: Variables assessed in the meal and emotion protocolling function of Jourvie Research

- Prepublication history and additional material for this paper are available online. To view these files, please visit the journal online (http://dx.doi. org/10.1136/bmjopen-2017018049).

Received 2 June 2017 Revised 21 September 2017 Accepted 28 September 2017

\section{CrossMark}

Department of Child and Adolescent Psychiatry, University Medicine Mainz, Mainz, Germany

\section{Correspondence to} Dr David R Kolar; david.kolar@unimedizin-mainz. de

\section{ABSTRACT}

Introduction As smartphones are widely distributed nowadays, mental health apps seem to be a promising treatment tool. First self-help apps for eating disorders have been developed recently. However, studies assessing the efficacy of such apps are scarce. A smartphone app could prevent further weight reduction and increase commitment during waiting time for outpatient treatment, especially for adolescents with anorexia nervosa (AN). In this study protocol, a randomised controlled trial to assess the efficacy of a smartphone-enhanced low-threshold intervention for AN during waiting time is described. Methods and analysis 30 adolescents with AN aged 12-19 years will be recruited at three child and adolescent psychiatry centres in Germany. All participants will be randomised to consultations only or consultations and the use of the Jourvie Research app. The app will be installed either on their own smartphone or on a research device. The participants will receive biweekly to monthly consultations for 3 months to review meal plans and weight management with a clinician. In addition, the Jourvie Research app for meal, behaviour and emotion protocolling will be provided to the intervention group. The protocols will be discussed with a clinician during the consultations. Dialectical behaviour therapy-informed skills for tension regulation to increase compliance with the meal plan will be taught in the intervention group and the app will remind the participant of a skill in a moment of need. The primary outcome is the age-adjusted and height-adjusted weight gain in standard deviation score after 3 months.

Ethics and dissemination Results will be disseminated at conferences and through peer-reviewed publications. The trial was approved by the ethics review board of the local medical association, Mainz, Germany, under the reference number 837.338 .15 .

Trial registration number German clinical trials register, reference number DRKS00008946.

\section{INTRODUCTION}

Anorexia nervosa (AN) is a severe eating disorder associated with a high impact on quality of life, significant health risks and
Strengths and limitations of this study

- The SELTIAN trial is the first randomised controlled trial to assess the efficacy of a low-threshold intervention enhanced with a smartphone app for adolescents with anorexia nervosa.

- A strength is the combination of face-to-face consultations with a smartphone app instead of distributing a smartphone app only.

- A considerable limitation is that the smartphone app relies on user input for presenting an ecological momentary intervention rather than on sensor data (eg, increased physical activity or heart rate measured by wearable sensors).

one of the highest mortality rates for mental disorders. ${ }^{1}{ }^{2}$ Lower body mass indices at first admission to mental health services are related to poorer treatment outcome and higher mortality, ${ }^{34}$ whereas a higher rate of weight gain during treatment is a predictor for recovery. ${ }^{5}$ Therefore, weight restoration of the patients is the main focus of early treatment stages.

Many evidence-based cognitive treatment approaches use self-monitoring to assess disordered eating behaviours in daily life. Self-monitoring is helpful in identifying emotional and behavioural factors which prevent weight gain and maintain disordered eating behaviour. ${ }^{67}$ Additionally, self-monitoring eating behaviour and mood fluctuations might increase insight into the disorder by the patient and concurrently lead to a faster weight gain.

With the wide distribution of smartphones nowadays, a powerful tool for assessment and intervention is available to most of adolescents. So-called mobile health applications (mhealth apps) for various mental disorders already exist and several have been shown as effective, for example, in the treatment of 
depression ${ }^{89}$ or anxiety. ${ }^{10}{ }^{11}$ Regarding mhealth apps for eating disorders, two recent reviews identified existing apps in the most prominent app stores and assessed the quality and clinical utility of these apps. ${ }^{12}{ }^{13}$ Several apps rely mostly on ecological momentary assessment (EMA) tools such as meal and emotion protocols, whereas only few apps allowed a direct data exchange with a therapist. Hence, in most cases, the interpretation of the observed behaviour and emotions is solely conducted by the user based on the feedback of the app, which might lead to underestimation of the severity of problematic behaviour. Surprisingly, none of the apps reviewed by Juarascio et $a l^{12}$ took advantage of the possibilities of ecological momentary interventions (EMI), which refers to delivering a psychosocial intervention immediately at the moment of need based on user input or sensor data. A third review analysing technology-based psychological interventions for eating disorders found preliminary evidence for the efficacy of mobile interventions (mostly text-message-based interventions) in the treatment of eating disorders. ${ }^{14}$ With respect to other forms of e-therapy for eating disorders (eg, CBT-E informed internet interventions), a meta-analysis found small beneficial effects but statistical confidence in the estimates was moderate to low. ${ }^{15}$ The included studies, however focused primarily on binge eating disorder and bulimia nervosa and its results might not be applicable to e-therapy for AN. Recently, an article on the development of one of the apps discussed in the mobile app reviews above was published, highlighting the good acceptance rates of the app as demonstrated in the utilisation behaviour in an international sample based on free downloads. ${ }^{16}$ Eightynine per cent of the users used the app at least once and the majority for $>30$ meal protocols. However, the efficacy of the app compared with other self-help tools was not assessed. In conclusion, all reviews identified a lack of controlled studies assessing the feasibility, efficacy and safety of smartphone apps for eating disorders.

\section{Rationale for a dialectical behaviour therapy-informed mhealth app for AN}

Emotion dysregulation is one of the key features for the development and maintenance of AN. ${ }^{17}{ }^{18}$ There is a growing body of evidence that highlights a causal connection between momentary levels of affect and disordered eating behaviour in individuals with AN. ${ }^{19}$ For instance, momentary emotional instability and negative urgency (ie, the tendency to immediately terminate states of negative affect) were found to be in direct relation to the occurrence of restrictive eating and active weight reduction in a study on adult individuals with AN. ${ }^{20}{ }^{21}$ Engel et $a t^{22}$ found that adults with AN showed more negative affect prior to and more positive affect after restrictive eating, which indicates that restrictive eating might be used as an emotion regulation strategy. However, monitoring mood and behaviour with an EMA approach did not substantially increase therapy outcome in a study on binge-eating disorder. ${ }^{23}$ With the technical possibilities of smartphones nowadays, modern mhealth apps might be used as a monitoring tool and offer specific interventions in the moment of need, which could increase the efficacy of such an app.

In a recent study on adolescents with $\mathrm{AN}$, higher levels of aversive tension were associated with food intake. ${ }^{24}$ Aversive tension is defined as an unpleasant state of high arousal, generally not associated with a specific emotion and with an action tendency towards immediate termination. ${ }^{25}{ }^{26}$ In the treatment of borderline personality disorder and non-suicidal self-harm of adults and adolescents, the dialectical behaviour therapy (DBT) approach has been shown to effectively reduce states of high aversive tension by teaching distress tolerance skills. ${ }^{27-29}$ DBT is a modern cognitive behavioural psychotherapy focusing on increasing emotion regulation capabilities of patients to reduce reactivity to distress. In DBT, it is assumed that patients with emotion dysregulation are doing their best but lack specific skills to effectively regulate their emotional states. ${ }^{30}$ As recommended in the most recent DBT skills training manuals, ${ }^{30}{ }^{31}$ skills should be taught and trained in the therapy session and an individual list of helpful skills should be created together with the patient. Training in these skills instead of only presenting a list of potentially helpful skills to the patient is considered to increase the perceived efficacy of the skills. Regarding DBT for eating disorders, recent trials have shown promising effects in the reduction of disordered eating behaviour and body mass index (BMI) increase in individuals with AN. ${ }^{32-34}$ A German randomised controlled trial (RCT) study comparing cognitive behavioural therapy and DBT with a control group was able to show that DBT was effective in the outpatient treatment of adolescents with AN. ${ }^{35}$ Thus, a DBT-informed skills module might be effective in reducing aversive tension during meal times and might subsequently increase calorie intake, when integrated into a smartphone app. A smartphone app could even propose a previously defined skill in the time of need, which would be of additional benefit.

Another important concern regarding smartphone apps for eating disorders is how such apps should be integrated in existing treatment. ${ }^{12}$ Currently, several mhealth apps are available in the app stores and can be used without prior or accompanying consultations. Especially in the case of eating disorders, thorough assessment of severity and comorbidity is crucial to prevent mortality and a chronic course of the disorder, which remains a challenge for many emerging mhealth solutions. ${ }^{36}$ In addition, there is evidence that guided technology-based psychological interventions for eating disorders, even if guidance of an experienced clinician is only applied on an email or text message basis, is perceived as supportive by most of the users. However, studies comparing guided and unguided (self-help) interventions have found mixed results regarding efficacy. ${ }^{14}$ Hence, we defined the following specifications for a DBT-informed, smartphone-based mhealth intervention for AN to facilitate weight restoration, normalisation of food intake and 
reduction of aversive tension and psychological distress during waiting time for outpatient treatment:

1. EMA of mood, meal and behaviour to assess momentary emotion dysregulation.

2. DBT-informed EMI module to deliver individual skills for reduction of aversive tension.

3. Training of individual EMI skills before presentation in the app to increase efficacy and compliance.

4. Guided app use and assessment of the protocols together with an experienced clinician to improve meal plans and decrease disordered eating.

In this trial, the efficacy of a smartphone app developed following the above-mentioned specifications will be tested in a group of adolescents with AN.

\section{Aims and hypotheses}

The aim of this study is to assess the efficacy of a smartphone app in combination with psychiatric consultations during the waiting time for outpatient psychotherapy for AN in comparison with psychiatric consultations only. An important factor in achieving a favourable outcome in AN is early weight gain. ${ }^{34}$ Hence, any intervention for AN during waiting time for outpatient treatment should focus on increasing the BMI SD score adjusted for age and height (BMI-SDS) of the patients rather than focusing on reduction of psychopathology only. Therefore, BMI-SDS was chosen as the primary outcome in this study.

We hypothesise that (1) after 3 months the height and age-adjusted weight gain of the participants receiving the additional smartphone app will be greater when compared with the participants receiving psychiatric consultations only (differences in BMI-SDS pretreatment to post-treatment). Moreover, we expect that (2) mean levels of daily aversive tension will be reduced significantly from baseline to closure in the intervention group compared with control group (mean aversive tension as measured in the EMA pretreatment and post-treatment). In addition, we will assess eating disorder psychopathology, depressiveness and general psychological distress at baseline and closure, as well as adherence to the protocolling scheme (eg, number of protocols recorded, number of sessions) and usability of the app as secondary outcomes.

\section{METHODS AND ANALYSIS \\ Trial design}

This study protocol describes a multicentre, two-arm RCT assessing the efficacy of a specific smartphone application in addition to supportive psychiatric consultations for adolescents with AN waiting for outpatient therapy. After informed consent is provided by the participant and her legal guardians in case of minority, the participant will be randomly assigned to either the smartphone-enhanced treatment condition (psychiatric consultations+specificmhealth app) or the treatment as usual condition (psychiatric consultations only). Each participant will receive a consultation every 2 weeks within the first 6 weeks after enrolment and further consultations when needed at least on a monthly basis throughout participation. Participants will be recruited at three different outpatient child and adolescent mental health services in Berlin, Mainz and Neuwied, Germany. Data will be collected at baseline, during the intervention and at the end of the intervention.

\section{Sample size calculation}

To our knowledge, there is no literature on smartphone-enhanced psychiatric consultations published. In a study on psychotherapy for AN, the waiting control group received $30 \mathrm{~min}$ psychiatric consultations every 2 weeks for 3 months with no effect on the BMI. ${ }^{35}$ In a previous study on guided self-help, a medium increase in BMI was found after 3 weeks of guided self-help. ${ }^{37}$ Therefore, we assume that single psychiatric consultations have no effect on the BMI and expect similar effects on the BMI in the smartphone-enhanced intervention as in guided self-help.

In this study, a repeated measurements analysis of variance (rmANOVA) assessing a within-between interaction of time and group will be used to test baseline to post-treatment BMI-SDS differences. A sample size of 22 participants is required to detect a medium effect size of $\mathrm{f}=0.25$ with a power of at least $80 \%$, an assumed correlation of $\mathrm{r}=0.70$ between measurements and an alpha-error level of $\propto=0.05$. Sample size calculations were conducted with $\mathrm{G}^{*}$ Power $^{38}$ and supervised by a biometrician. In order to reach at least 22 complete data sets, 30 participants are planned to be enrolled in the study, which will create a reasonable margin for possible dropouts.

\section{Recruitment and participants}

Recruitment will be conducted at the study centres. Prior to the study, general practitioners, paediatricians (child and adolescent) psychiatrists and psychotherapists in private practice in close distance to the study centres will be informed of the study and referral options by a personal letter.

Due to ethical reasons, we decided to include participants currently waiting for outpatient treatment. In Germany, waiting times for outpatient psychotherapy are at 12.5 weeks on average. ${ }^{39}$ Especially patients with AN might wait even longer as this disorder is considered hard-to-treat and many psychotherapists refuse to treat adolescents with AN in an outpatient setting.

Female participants aged 12-19 years who have been diagnosed with either full syndrome or atypical AN according to ICD-10 at one of the study centres will be eligible for participation. Clinical diagnoses will be confirmed by conducting the Eating Disorder Examination (EDE) in the adult or children version, a structured interview for the valid and reliable assessment of eating disorders. ${ }^{4041}$ In addition, amenorrhoea will be assessed to meet ICD-10 criteria for AN. ${ }^{42}$ As males and females differ regarding the prevalence ${ }^{43}$ and psychopathology of $\mathrm{AN},{ }^{44}$ only females will be included to prevent gender effects on the primary outcome. Patients who receive outpatient 
psychotherapy on a regular basis (ie, at least weekly 50 min sessions with a licensed psychotherapist) will not qualify for inclusion. However, preparatory sessions (up to five initial sessions with a licensed psychotherapist for diagnostic and goal establishing purposes) during participation will be encouraged to facilitate prompt outpatient treatment after completion of the study. Pharmacological treatment of comorbid disorders (eg, depression) will be permitted during participation. Patients with a comorbid personality disorder of the emotionally unstable subtype will be excluded, as this condition is related to heightened levels of aversive tension, ${ }^{26}$ which is a secondary outcome in this study. Patients with severe suicidal ideation will not be eligible for participation as inpatient treatment is recommended for safety reasons. Participants who will be referred to inpatient treatment for $>1$ week (ie, mostly due to severe somatic complications or inpatient refeeding) during participation will be considered as dropouts.

All participants and their legal guardians will be made aware of the voluntary nature of the study. Refusing to participate will not affect the treatment of the participant in any way. Furthermore, both participants and their legal guardians in case of minority will be required to consent to participation after being provided with extensive information on the study by the local study coordinator. All participants will be required to install the smartphone application on their private smartphone or to agree with using a smartphone distributed by the study centre. The first participant was recruited in April 2016. Recruitment is expected to close in 2018.

\section{Randomisation}

Following baseline assessment, participants will be randomised to either the smartphone-enhanced intervention arm or the treatment-as-usual condition with a 3:2 allocation rate. Randomisation will be stratified for the study centres and the ICD-10 subtypes of AN (restrictive, binge-purge, atypical) to prevent possible confounding effects by baseline weight and psychopathology differences of AN subtypes and potential structural differences of the study centres (two university medical centres and one public child and adolescent psychiatry hospital). The randomisation procedure will be conducted on a web-based platform using REDCap. ${ }^{45}$ Allocation tables are concealed within REDCap. Randomisation will be conducted by the study coordinator at each study centre. Due to the nature of the study, neither blinding of the counsellors nor the participants can be conducted. With an allocation ratio of 3:2 for the intervention group, 18 participants in the intervention group and 12 participants in the control group will be included. However, as randomisation is stratified, the final distribution of the groups might differ from the 3:2 ratio.

\section{The Jourvie Research app}

In 2015, Jourvie gUG (Berlin, Germany) released an Android and iOS app for self-help and therapy enhancement of patients with eating disorders. ${ }^{46}$ The freely available app provides users with an electronic meal, behaviour and emotion protocol function, and the opportunity to browse and enter coping strategies. The app assesses the 'when, where, what, with whom' of any meal the user entered, asks if the user was hungry and if she felt sated after the meal. Additionally, the intensity of several emotions and the urge to or actually engage in maladaptive emotion regulation behaviours (eg, bingeing, purging, self-harm) is assessed at every data entry. Users can specify times for automatic reminders to fill in the meal protocol. The data can be shared by email with the user's personal therapist. The growing number of users as well as their positive feedback shows the high acceptance rate of the original Jourvie app.

For the SELTIAN study, we enhanced the Jourvie app by implementing an additional EMI module. In this Jourvie Research version for Android smartphones, the EMA part consists of the standard meal and emotion protocol of the Jourvie app. Furthermore, a visual analogue slider was added to assess current aversive tension from 0 to 100 , as the EMI part of the application will be triggered by the intensity of aversive tension at the time data are entered. If a value of aversive tension $>50$ and $<70$ is entered, the app will advise the user to use an individual

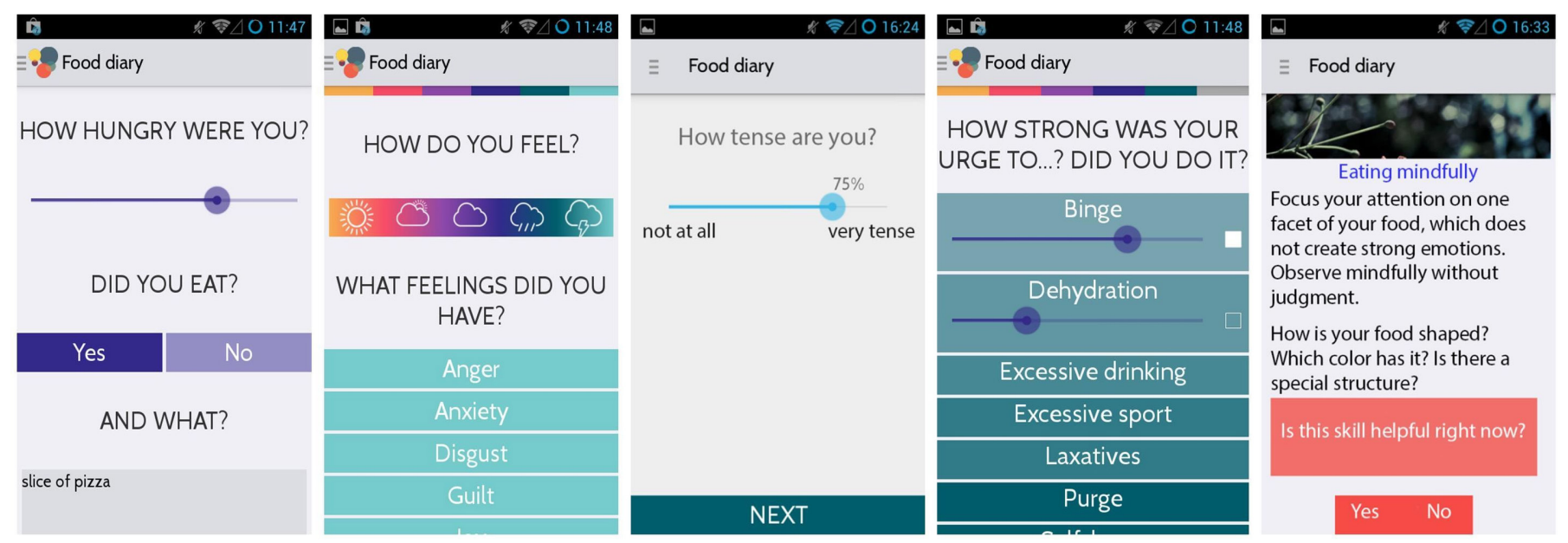

Figure 1 Screenshots of the Jourvie Research application as used in the intervention group. 
skill for reduction of aversive tension and to facilitate a regular eating behaviour. If a value of aversive tension $>70$ is entered, the app shows a short instruction for an individual skill of the high distress category. The skills will be trained in the intervention sessions and a short instruction on how to apply this particular skill will be uploaded automatically onto the smartphone of the participant once the counsellor has entered the skill instruction into the server database. Figure 1 shows screenshots of the Jourvie Research EMA and EMI modules.

Each meal and emotion protocol will be uploaded immediately after completion to a server of the University Medical Centre of the Johannes Gutenberg University Mainz, Germany. The counsellors will have online access to the individual protocols of their participants at any point in time and can print the protocol before the consultations to review them with the participants. The connection to the server database will be protected by SSL encryption. After uploading the data, the local copy of the meal and emotion protocol on the smartphone will be deleted for data safety reasons.

\section{Treatment arms}

After randomisation, participants will be enrolled in one of two treatment arms. For 3 months, participants will receive psychiatric sessions by a counsellor with a master's degree in psychology, a medical doctor or equivalent. A child and adolescent psychiatrist or a licensed psychological psychotherapist experienced with DBT will supervise the counsellors. Ideally, each patient will receive all sessions from the same counsellor. All counsellors will receive a thorough explanation on how to obtain individual protocols from the database and how to upload skill instructions onto the smartphones of the participants.

Figure 2 shows the study flow diagram and gives an overview of the treatment arms. In each treatment arm, three standardised sessions will be held in the first 6 weeks of the intervention. Each session should last

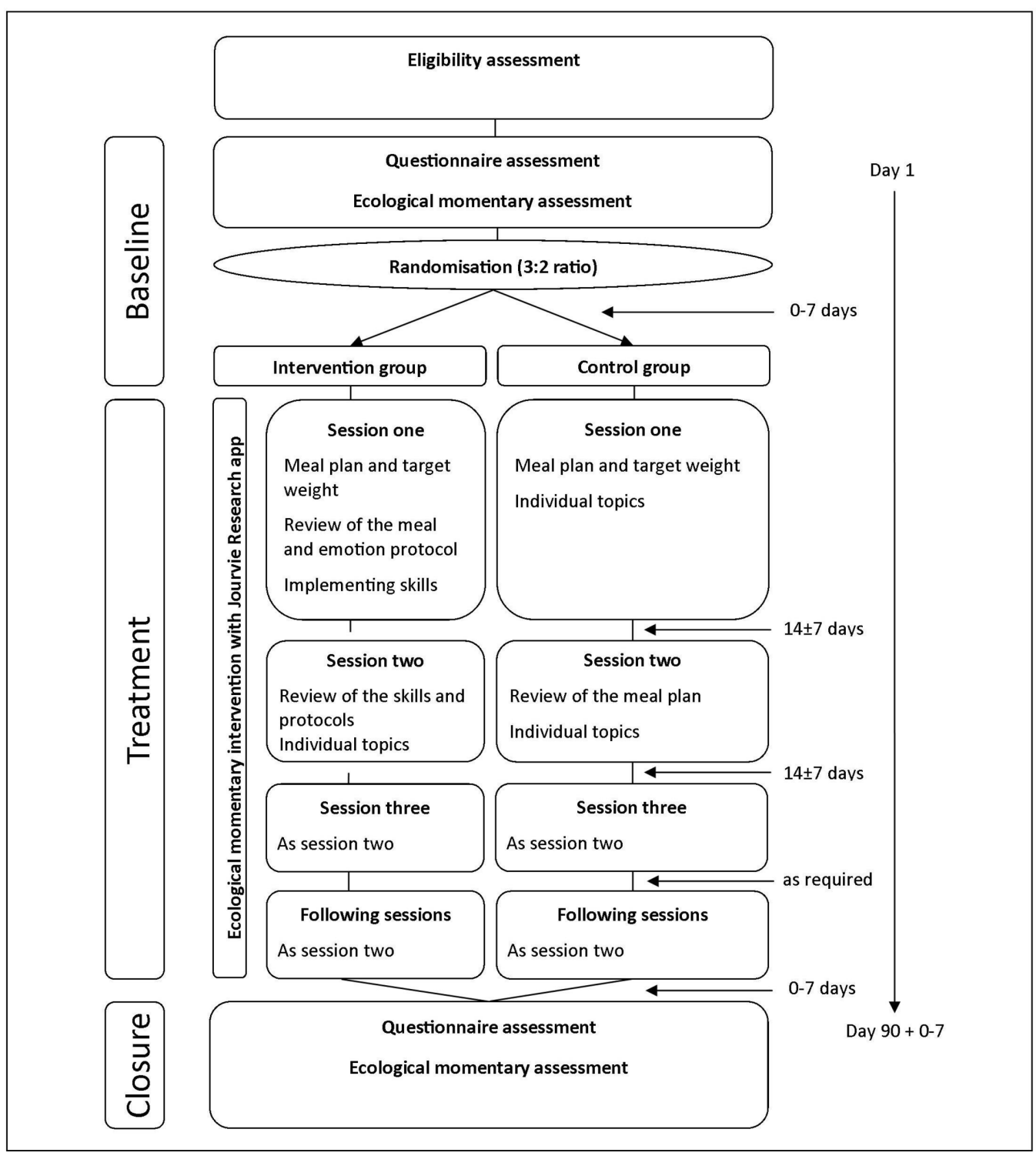

Figure 2 Study flow chart. 
$50 \mathrm{~min}$, depending on the needs of the participant and her parents. After the initial 6-week phase, participants will receive follow-up sessions on at least a monthly basis, depending on the severity of the disorder and the initial weight gain in the first 6 weeks. At each session, the participant will be weighed in underwear.

\section{Control condition}

In the control condition, a meal plan and a target weight will be established with the participant during the first session. Both the participants and their legal guardians should commit themselves to the meal plan and the target weight. The target weight should be achievable within 3 months with a weekly weight gain of $200-500 \mathrm{~g}$ in accordance with national guidelines. ${ }^{47}$ Special focus will be set on individual factors maintaining the restrictive eating and low body weight of the participant. In the second and third session, the weight gain or loss of the participant will be assessed and reviewed in the session. The counsellor will guide the participant in developing strategies to further continue or to achieve weight gain by adapting the meal plan. Unlike the smartphone-enhanced treatment condition, these strategies will focus on a 'treatment as usual approach' to increase weight rather than on reducing aversive tension. Individual topics can be discussed in addition. The following sessions will be similar to the second session in a frequency depending on the individual need of the participant.

\section{Smartphone-enhanced treatment}

In the smartphone-enhanced treatment condition, the participants will install the Jourvie Research app on their private Android smartphone or receive a prepared Android smartphone for the duration of the study. In the first session, a meal plan and a target weight will be established with the participant and their legal guardians if applicable, similar as in the control condition. Additionally, the participant will be introduced to the skill instruction function of the application. 'Skills' in this context are DBT-informed emotion regulation techniques, which aim at reducing aversive tension primarily, as restrictive eating is considered a maladaptive emotion regulation strategy to reduce aversive tension. ${ }^{18}{ }^{24}$ A first skill to facilitate regular eating behaviour during meal times will be taught and discussed with the participant and finally a short description of the skill will be entered into the application, if necessary. The skills can be either individualised (eg, the participant states that listening to her favourite song helps her in eating a whole meal) or taken from a list of helpful skills given to all counsellors in the study. The skills on this list are derived from the emotion regulation, mindfulness and stress tolerance chapters of the DBT skills manuals for adults and adolescents ${ }^{48} 49$ and grouped into two categories (skills for aversive tension of 50-70 and skills for aversive tension $>70$ ). The participant will be advised to fill in the meal and emotion protocols thoroughly until the next session and to make use of the skill whenever necessary or recommended by the application.

In the second session, the counsellor will evaluate the meal and emotion protocol together with the participant to identify particular situations in which disordered eating or compensatory behaviour occurred. Furthermore, the counsellor will focus on the use of the skills taught in the last session and modify the ones already known or teach new skills in relation to the specific problem behaviour which impedes weight gain.

During the third session, the meal and emotion protocol will be reviewed again and adjustments to the meal plan and/or the individual skills list will be made. If time is available, individual topics can be discussed. The following sessions will be similar to the third session, always starting with a review of the protocol, adjusting the individual skills if necessary and allowing time for individual topics.

\section{Outcome measures}

In this study, data will be assessed at several points in time during the intervention. Table 1 shows the schedule of assessments.

\section{Weight and height measurement}

All patients will be weighed in underwear on a calibrated scale each time before a session by a trained female student assistant or nurse. At inclusion and closure of the study, height will also be measured. With these measurements, the BMI at each time point will be calculated as a measure of weight gain. As only baseline- BMI and closure-BMI will be used as primary outcome measures, height will only be assessed at the first and last measurement for practicability reasons. Both in adolescent and severely underweight populations, the BMI-SDS is a better measurement than the BMI as it accounts for age-specific and gender-specific effects on BMI. The BMI-SDS values provided by Kromeyer-Hauschild et a $\tilde{l}^{0}$ will be used for adolescent participants and the BMI-SDS values of the Second National Nutrition Study for participants older than 18 years. ${ }^{51}$

\section{Questionnaire assessment}

At both inclusion and closure, several questionnaires will be administered and filled in by the participants. All questionnaires are self-completed electronically in the data management software REDCap. ${ }^{45}$

\section{Eating Disorder Inventory-2}

The Eating Disorder Inventory-2 (EDI-2) ${ }^{52}$ is an instrument to assess eating disorder-specific psychopathology with a dimensional approach. The German version showed overall acceptable psychometric properties in the general population and good properties in an eating disorder group (internal consistency from $\alpha=0.66$ to $\alpha=0.94$ )$^{53}$ The convergent validity with other eating disorder measurements was good. In this study, the short form assessing eight subscales will be used (drive for thinness, bulimia, perfectionism, ineffectiveness, interpersonal 
Table 1 Assessment schedule

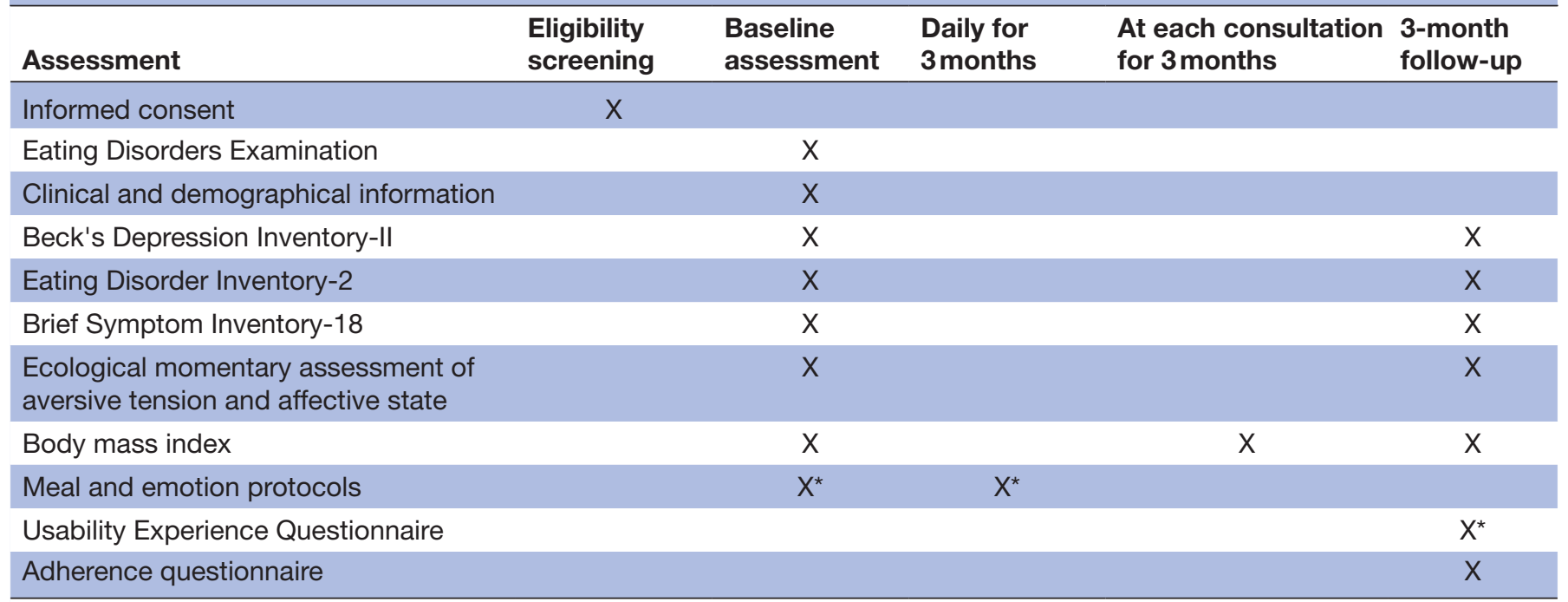

*Will be assessed in the intervention group only.

distrust, body dissatisfaction, interoceptive awareness, maturity fear). The EDI-2 will be used as a dimensional measure of eating disorder psychopathology in this study.

\section{Beck's Depression Inventory-II}

Beck's Depression Inventory-II (BDI-II) ${ }^{54}$ is one of the most widely used self-report instruments to assess depressive symptoms. The German version ${ }^{55}$ showed good psychometric properties when applied to adolescents (Cronbach's alpha $\alpha=0.94$ ) and is recommended as a screening instrument for depression as it discriminates sufficiently between patients and healthy control participants (Cohen's $d=2.32) .{ }^{56}$ In this study, the BDI-II will be used as a dimensional measure of depressiveness.

\section{Brief Symptom Inventory-18}

The Brief Symptom Inventory-18 (BSI-18) is a short scale assessing an overall severity index of psychological symptoms. ${ }^{57}$ The inventory consists of three six-item scales (somatisation, depression, anxiety) of the longer Derogatis Symptom Checklist 90R. ${ }^{58}$ Factor analysis confirmed the three-factor structure. The BSI-18 showed good reliability (Cronbach's alpha $\propto=0.91$ for the global symptom index) and validity. The global severity index of the inventory will be used as a general psychological distress measure.

\section{User Experience Questionnaire}

The User Experience Questionnaire (UEQ) is a questionnaire primarily developed for measuring the usability of websites. ${ }^{59}$ With this questionnaire, the user experience of a website is assessed on various dimensions on a 7-point bipolar scale (eg, if an application is rather attractive than unattractive, more creative than dull). The UEQ contains six subscales of different facets of user experience. Internal consistencies (Cronbach's $\alpha$ ) for the German version of the subscales are between $\alpha=0.73$ and $\alpha=0.89$ when applied to rate a website. As this questionnaire is one of the few reliable and valid instruments to assess user experience, we used the questionnaire to rate a mobile application, in line with a previous study. ${ }^{60}$ However, it is not validated for assessing mobile apps and results must be considered carefully. Only participants of the intervention group will fill in this questionnaire at the 3-month-follow-up.

\section{Adherence questionnaire}

At closure, all participants will answer several questions regarding their experience of and adherence to the treatment, for example, if they used other mobile apps for eating disorders during participation, using a 7-point Likert-scale. In addition, participants in the treatment group will be asked if they found the smartphone application to be useful and if they would recommend it to other adolescents with AN. Finally, the participants will be asked to give written qualitative feedback on the study.

\section{Ecological momentary assessment}

At baseline and for the 3-month-follow-up, all participants will conduct a 24hours EMA for the measurement of aversive tension and other affective variables. Therefore, the Android application movisensXS in its latest version (movisens GmbH, Karlsruhe, Germany) will be installed on the participants' smartphones. Within movisensXS, a short questionnaire will be prompted every hour. During sleep times, patients can turn off their smartphone. Items of the EMA can be found in table 2. Two items regarding aversive tension and emotion identification will be used, as in previous studies on these variables in an EMA on adolescents with $\mathrm{AN} .{ }^{24}$ With respect to affective variables, a shortened version of the Positive and Negative Affective Schedule $^{61}$ will be used, as in previous studies on affective states in adults with $\mathrm{AN} .^{22}$ The German translation of these items will be in line with Krohne et al. ${ }^{62}$ The EMA data on aversive tension will be used for measuring 
Table 2 Items of the ecological momentary assessment conducted at pretreatment and post-treatment

\begin{tabular}{|c|c|c|}
\hline$\#$ & Item & Answer format \\
\hline 1 & $\begin{array}{l}\text { On a scale from } 0 \text { to } 100 \text {, which level of aversive tension are you } \\
\text { feeling right now? }\end{array}$ & Visual analogue scale (0-100) \\
\hline 3 & $\begin{array}{l}\text { On a scale from (0 to 100), how sure are you of what you are feeling } \\
\text { right now? }\end{array}$ & Visual analogue scale $(0-100)$ \\
\hline
\end{tabular}

${ }^{*}$ Derived from the original PANAS Scale ${ }^{61}$ according to Engel et al, ${ }^{22}$ containing the following items: nervous, disgusted, distressed, ashamed, angry at self, afraid, sad, dissatisfied with self, strong, enthusiastic, proud, attentive, happy, energetic, confident and cheerful.

mean aversive tension throughout the day at baseline and closure of the study in both groups.

\section{Jourvie Research assessment (smartphone-enhanced treatment group only)}

Participants in the smartphone-enhanced treatment group will be required to record their meals and emotions frequently by using the Jourvie Research app. Per default, each participant will be reminded three times during the day (at 08:00, 13:00, 19:00) to complete the protocol. Participants will be able to adjust the reminders to their individual meal times or even turn them off. Additionally, individual data entry will be possible at any given time. Depending on whether the user will have eaten before, the participant will be required to fill in a complete meal and emotion protocol or just to record the current level of aversive tension. Online supplementary one describes the variables recorded with the Jourvie Research app.

\section{Session assessment}

For each session, the therapist will fill in a short questionnaire in our electronic data management system. The day as well as the start and end time of each session will be recorded. Additionally, the therapist is required to briefly describe the topics of the session. In the smartphone-enhanced treatment group, the introduction and deletion of skills will be monitored as well. Therefore, the number and duration of sessions within each group will be comparable as a potential influencing factor.

\section{Dropout assessment}

In case of a dropout, the reason for the dropout and the weight and height at the time of dropout will be assessed, if applicable.

\section{Data management and data safety}

Study data will be collected and managed using REDCap electronic data capture tools hosted at University Medical Centre of the Johannes Gutenberg University, Mainz, Germany. ${ }^{45}$ SSL encryption will be used during data transfer. Once the transfer is completed, the local copy on the smartphone of the participant will be deleted. To increase data safety in case of loss or theft of the smartphone, the Jourvie Research app will be encrypted and secured with a private password only known to the participant. Personal data (ie, name and residence) will be stored only locally. All data stored on the University Medical Centre of the Johannes Gutenberg University Mainz or Movisens servers will be pseudonymised. However, participants will be made aware that when entering personal data into one of the protocol fields, anonymity cannot be guaranteed. The data management of this study was approved by the institutional data safety boards of the University Medical Centre of the Johannes Gutenberg University Mainz and the Charité University Medical Centre Berlin, Germany.

\section{Statistical analyses}

Data will be analysed using SPSS 23.0 software (SPSS, Chicago, Illinois, USA). As AN is a disorder which is difficult to treat, high dropout rates can be expected due to, for example, somatic complications and inpatient referral $\left(\mathrm{eg}, 22 \%\right.$ in the ANTOP study $\left.{ }^{63}\right)$. In addition, as this is a trial during waiting time, it is assumed that several participants will drop out of the treatment when they start regular outpatient psychotherapy within the 3 months duration of the study. Therefore, the primary outcome will be analysed by intention-to-treat with a last-observation-carried-forward procedure (ie, all participants who completed baseline, randomisation and at least one consultation will be included in analysis). The primary outcome is the difference in the BMI-SDS prior to and after the treatment phase (90 days). A confirmatory rmANOVA will be conducted to compare the intervention and control group BMI-SDS differences with an alpha error level of $5 \%$. A second rmANOVA will be conducted to assess group differences regarding the average daily level of aversive tension before and after the treatment (secondary outcome). In addition, a thorough confounder analysis will be conducted to evaluate group differences regarding the number of sessions, comorbidity, dropout, inpatient treatment after completion of the study.

\section{Ethics and dissemination}

The study is registered at the German Clinical Trials Register under the reference number DRKS00008946. Participants or their legal guardians can withdraw from the study at any time without providing a reason for 
withdrawal and without any consequences for ongoing or future treatment. For medical safety, participants' weight and psychopathology as well as potential adverse events will be closely monitored by a medical supervisor. Severe adverse events will be reported to the local ethics committee. Participants who continue to wait for treatment after completion of the study will be provided with additional sessions until regular treatment is started. The head of the Department of Child and Adolescent Psychiatry at the University Medical Centre of the Johannes Gutenberg University Mainz can terminate the study at any time for safety reasons. Parts of an early version of the study protocol were presented as a poster at the Eating Disorders International Conference 2016, London, UK, and at the congress of the German Society for Eating Disorders 2016, Essen, Germany. ${ }^{64}$ Results of this trial will be disseminated as publications in peer-reviewed journals and presented at conferences.

\section{DISCUSSION}

In this study protocol, we present the first RCT to assess the efficacy of a low-threshold psychological intervention enhanced with a DBT-informed mhealth app on smartphones for patients with AN waiting for outpatient psychotherapy. Two recent reviews of the literature on smartphone apps for eating disorders could not identify any RCTs testing the outcome of these apps. ${ }^{12}{ }^{13}$ Our expectations are that using Jourvie Research as a monitoring and intervention app in a guided setting will increase BMI-SDS and decrease aversive tension of the participants significantly during the waiting time for treatment. However, we do not expect that a smartphone app will render psychotherapy obsolete.

A notable strength of the study is that it allows a direct intervention in a naturalistic setting (ie, a skill provided exactly when needed). The participant enter valuable data for the assessment in the next session, and the therapist can provide the participant with new skills depending on feedback from previously tested skills. This might be essential for behavioural change, as an earlier study on patients with binge-eating disorder using EMA as a treatment tool did not find any effects on behaviour. ${ }^{23}$ In particular, time-congruent recording of aversive tension and the presentation of skills may have a beneficial effect on adolescents when they use a smartphone in contrast to paper and pencil approaches. In addition, electronically recording meal protocols might reduce stigmatising conditions compared with filling in a paper-based protocol during lunch in a restaurant. Comparing our trial with other EMA studies in the field of eating disorders, the possibility to use the participants' own smartphone for data capture and intervention might offer more accurate and complete data, especially of adolescents.

A considerable limitation is that Jourvie Research relies on user input for triggering EMIs. Taking advantage of the sensor data of a smartphone might increase chances that the intervention is presented in the moment of need.
However, the reliability of sensor data (eg, of the smartphone itself or wearables such as smartwatches, activity trackers) has to be tested before integration. Due to the automatic trigger of an intervention, the skill presented in Jourvie research might not be applicable in the given situation for the participant (eg, a skill using ice cubes might not be applicable during school). We tried to minimise these difficulties by offering skills which are in general applicable during meals and do not rely on additional tools (eg, ice cubes, chili peppers).

Another limitation is that the number of psychiatric consultations with a clinician is allowed to vary between the participants. Adolescents with AN require different amounts of care depending on the current physiological health, motivation to change and the current BMI. A flexible consultation schedule accounts for these needs and increases ecological validity of the study. However, this degree of freedom might confound the results, if a systematic group effect regarding the number of consultations or a significant correlation of consultations with the BMI-SDS occurs. To minimise a systematic effect, the number of consultations is fixed in the first 6 weeks (comparable to a training phase) and only based on the individual needs of the participant in the remaining time. On a statistical level, group differences regarding the number of consultations will be assessed and included into the analyses as a covariate if systematic group differences occurred. Finally, assessing the direct influence of the smartphone application on the primary outcome might be difficult, as the intervention and control treatments differ on several levels. However, the impact of the smartphone application can be estimated by assessing whether a high number of completed protocols (measuring the use of the app) is correlated with any of the outcome measures.

Several ethical concerns need to be discussed when an EMI is conducted. First of all, participants can only agree to the treatment on a general level, as interventions will be triggered automatically, even if the participant does not explicitly wish to receive any intervention in this specific moment. However, we assume that by allowing participants to dismiss a certain intervention or report the intervention as 'not helpful', the participant has sufficient control over the intervention. Second, by using smartphones and internet connections, data safety must be guaranteed as far as possible. To maximise the safety of the health data of our participants, we rely on encrypted data transfer and data storage on the smartphones of the participants. In addition, every data entry will be deleted on the participants' smartphone once the data were successfully transferred to the servers of our research department. Hence, the risk that health data can be read in case of theft of the smartphone and decryption of the Jourvie Research app is reduced to a minimum.

Considering adverse effects in mhealth or smartphone treatments for eating disorders, several potential risks are conceivable: there is a potential risk that monitoring food intake might increase restrictive eating when participants are confronted with a subjectively too large amount of food intake during their day which otherwise would have 
remained unnoticed. This is of particular concern as any meal protocolling app can be used as a weight loss app when assessing whether food intake was reduced to the previous day. In addition, $\mathrm{AN}$ is often associated with perseverance and perfectionism. ${ }^{65}$ Participants with high levels of these personality traits might not benefit of a meal protocol app as they might focus more rigidly on controlling and protocolling their meals and behaviour than on changing their behaviour based on the protocols. To minimise this risk, the app does not allow reviewing previous meal protocols, and calorie content of food intake is not assessed explicitly. Furthermore, all participants will review their protocols directly with a clinician to increase the motivation to change based on their previously protocolled behaviour.

A potential outcome of our study in favour of the intervention with the smartphone app might encourage researchers to investigate other interventions enhanced with smartphones for eating disorders and other mental disorders. Implementing the results of this trial into practice could result in better overall outpatient psychotherapy outcomes in AN if psychotherapy could focus on the underlying cognitive and emotional causes from the start rather than stabilising the weight of the patient during the first sessions. However, this remains speculative and needs to be evaluated once an implementation into practice is made.

In conclusion, the SELTIAN trial offers the opportunity to assess the efficacy of a low-threshold intervention enhanced with a smartphone app for adolescents with AN. If a positive effect of the intervention with the smartphone app on the $\mathrm{BMI}$ is found, assessing the efficacy of a smartphone app as a treatment add-on for outpatient treatment of $\mathrm{AN}$ and other eating disorders should be considered. Given that AN is a disorder which is hard to treat, especially in an outpatient setting with only low-frequent face-to-face sessions, it is hard to predict if our intervention will directly affect weight gain. However, we hope that apart from the primary aim of weight restoration, the evaluation of this first trial of a smartphone app will provide a basis to further studies on the efficacy, usability and safety of smartphone mhealth apps in the treatment of eating disorders.

Acknowledgements The authors are thankful to Betteke Van Noort and Claudia Thurn from Charité Berlin, Germany, for discussing an earlier version of the study protocol. The authors are further thankful to the team of Jourvie gUG (limited liability), Berlin, Germany, for developing and implementing our requirements into the Jourvie Research application. Melina Roth, Miriam Postulka and Karin Perthes participated in the coordination and psychological assessment of the study. Alexander Böpple conducted proof-reading. The authors are further thankful to all present and future participants of the SELTIAN trial.

Contributors DK conceived the study. DK, FH, EJ and MH participated in the design of the study. DK drafted the first version of the manuscript. FH and EJ revised the study protocol. All authors read and approved the final version of the manuscript.

Funding This work was supported by a research grant of the intramural research funding of the Johannes Gutenberg-University Mainz, Germany.

Competing interests None declared.

Ethics approval The trial was approved by the ethics review board of the local medical association, Mainz, Germany, under the reference number 837.338.15. In addition, the protocol was also approved by the institutional review board of the Charité University Medical Centre, Berlin, Germany, for the Berlin study centre.

Provenance and peer review Not commissioned; externally peer reviewed.
Open Access This is an Open Access article distributed in accordance with the Creative Commons Attribution Non Commercial (CC BY-NC 4.0) license, which permits others to distribute, remix, adapt, build upon this work non-commercially, and license their derivative works on different terms, provided the original work is properly cited and the use is non-commercial. See: http://creativecommons.org/ licenses/by-nc/4.0/

(C) Article author(s) (or their employer(s) unless otherwise stated in the text of the article) 2017. All rights reserved. No commercial use is permitted unless otherwise expressly granted.

\section{REFERENCES}

1. Zipfel S, Löwe B, Reas DL, et al. Long-term prognosis in anorexia nervosa: lessons from a 21 -year follow-up study. Lancet 2000;355:721-2.

2. Franko DL, Keshaviah A, Eddy KT, et al. A longitudinal investigation of mortality in anorexia nervosa and bulimia nervosa. Am J Psychiatry 2013;170:917-25.

3. Salbach-Andrae H, Schneider N, Seifert K, et al. Short-term outcome of anorexia nervosa in adolescents after inpatient treatment: a prospective study. Eur Child Adolesc Psychiatry 2009;18:701-4.

4. Steinhausen HC, Grigoroiu-Serbanescu M, Boyadjieva S, et al. The relevance of body weight in the medium-term to long-term course of adolescent anorexia nervosa. Findings from a multisite study. Int $J$ Eat Disord 2009;42:19-25.

5. Lund BC, Hernandez ER, Yates WR, et al. Rate of inpatient weight restoration predicts outcome in anorexia nervosa. Int $\mathrm{J}$ Eat Disord 2009;42:301-5.

6. Fairburn CG, Cooper Z, Shafran R, et al. Enhanced cognitive behavior therapy for eating disorders: the core protocol. In: Fairburn CG, ed. Cognitive behavior therapy and eating disorders. New York: Guilford Press, 2008:47-72.

7. Jacobi C, Thiel A, Beintner I. Anorexia und bulimia nervosa. Ein kognitiv-verhaltenstherapeutisches Behandlungsprogramm. Basel: Beltz, 2016.

8. Schmädeke S, Bischoff C. Wirkungen smartphonegestützter psychosomatischer Rehabilitationsnachsorge (eATROS) bei depressiven patienten. Verhaltenstherapie 2015;25:277-86.

9. Schlosser DA, Campellone TR, Truong B, et al. The feasibility, acceptability, and outcomes of PRIME-D: A novel mobile intervention treatment for depression. Depress Anxiety 2017;34:546-54.

10. Loo Gee B, Griffiths KM, Gulliver A. Effectiveness of mobile technologies delivering Ecological Momentary Interventions for stress and anxiety: a systematic review. J Am Med Inform Assoc 2016;23:221-9.

11. Mohr DC, Tomasino KN, Lattie EG, et al. IntelliCare: an eclectic, skills-based app suite for the treatment of depression and anxiety. J Med Internet Res 2017;19:e10.

12. Juarascio AS, Manasse SM, Goldstein SP, et al. Review of smartphone applications for the treatment of eating disorders. Eur Eat Disord Rev 2015;23:1-11.

13. Fairburn CG, Rothwell ER. Apps and eating disorders: a systematic clinical appraisal. Int J Eat Disord 2015;48:1038-46.

14. Schlegl S, Bürger C, Schmidt L, et al. The potential of technologybased psychological interventions for anorexia and bulimia nervosa: a systematic review and recommendations for future research. J Med Internet Res 2015;17:e85.

15. Loucas CE, Fairburn CG, Whittington C, et al. E-therapy in the treatment and prevention of eating disorders: A systematic review and meta-analysis. Behav Res Ther 2014;63:122-31.

16. Tregarthen JP, Lock J, Darcy AM. Development of a smartphone application for eating disorder self-monitoring. Int $J$ Eat Disord 2015;48:972-82.

17. Lavender JM, Wonderlich SA, Engel SG, et al. Dimensions of emotion dysregulation in anorexia nervosa and bulimia nervosa: a conceptual review of the empirical literature. Clin Psychol Rev 2015;40:111-22.

18. Haynos AF, Fruzzetti AE. Anorexia nervosa as a disorder of emotion dysregulation: evidence and treatment implications. Clin Psychol Sci Pract 2011;18:183-202.

19. Engel SG, Crosby RD, Thomas G, et al. Ecological momentary assessment in eating disorder and obesity research: a review of the recent literature. Curr Psychiatry Rep 2016;18:37.

20. Culbert KM, Lavender JM, Crosby RD, et al. Associations between negative affect and binge/purge behaviors in women with anorexia nervosa: Considering the role of negative urgency. Compr Psychiatry 2016;66:104-12.

21. Selby EA, Cornelius T, Fehling KB, et al. A perfect storm: examining the synergistic effects of negative and positive emotional instability 
on promoting weight loss activities in anorexia nervosa. Front Psychol 2015;6:1260.

22. Engel SG, Wonderlich SA, Crosby RD, et al. The role of affect in the maintenance of anorexia nervosa: evidence from a naturalistic assessment of momentary behaviors and emotion. J Abnorm Psychol 2013;122:709-19.

23. le Grange D, Gorin A, Dymek M, et al. Does ecological momentary assessment improve cognitive behavioural therapy for binge eating disorder? A pilot study. Eur Eat Disord Rev 2002;10:316-28.

24. Kolar DR, Hammerle F, Jenetzky E, et al. Aversive tension in female adolescents with Anorexia Nervosa: a controlled ecological momentary assessment using smartphones. BMC Psychiatry 2016;16:97

25. Stiglmayr CE, Bischkopf J, Albrecht V, et al. The experience of tension in patients with borderline personality disorder compared to other patient groups and healthy controls. J Soc Clin Psychol 2008;27:425-46.

26. Stiglmayr CE, Grathwol T, Linehan MM, et al. Aversive tension in patients with borderline personality disorder: a computer-based controlled field study. Acta Psychiatr Scand 2005;111:372-9.

27. Mehlum L, Tørmoen AJ, Ramberg M, et al. Dialectical behavior therapy for adolescents with repeated suicidal and self-harming behavior: a randomized trial. J Am Acad Child Adolesc Psychiatry 2014;53:1082-91.

28. McMain SF, Guimond T, Barnhart R, et al. A randomized trial of brief dialectical behaviour therapy skills training in suicidal patients suffering from borderline disorder. Acta Psychiatr Scand 2017;135:138-48.

29. Booth R, Keogh K, Doyle J, et al. Living through distress: a skills training group for reducing deliberate self-harm. Behav Cogn Psychother 2014;42:156-65.

30. Linehan M. DBT skills training manual. Guilford Publications, 2014.

31. von Auer AK, Bohus M. eds. Interaktives skillstraining für jugendliche mit problemen der gefüh/sregulation (DBT-A). Stuttgart: Schattauer, 2017.

32. Chen EY, Segal K, Weissman J, et al. Adapting dialectical behavior therapy for outpatient adult anorexia nervosa-a pilot study. Int J Eat Disord 2015;48:123-32.

33. Kröger C, Schweiger U, Sipos V, et al. Dialectical behaviour therapy and an added cognitive behavioural treatment module for eating disorders in women with borderline personality disorder and anorexia nervosa or bulimia nervosa who failed to respond to previous treatments. An open trial with a 15-month follow-up. J Behav Ther Exp Psychiatry 2010;41:381-8.

34. Federici A, Wisniewski L. An intensive DBT program for patients with multidiagnostic eating disorder presentations: a case series analysis. Int J Eat Disord 2013;46:322-31.

35. Salbach-Andrae H, Bohnekamp I, Bierbaum T, et al. Dialektisch Behaviorale Therapie (DBT) und Kognitiv Behaviorale Therapie (CBT) für Jugendliche mit Anorexia und Bulimia nervosa im Vergleich. Kindheit und Entwicklung 2009;18:180-90.

36. Aardoom JJ, Dingemans AE, Van Furth EF. E-Health Interventions for Eating Disorders: Emerging Findings, Issues, and Opportunities. Curr Psychiatry Rep 2016;18:42.

37. Cardi V, Clarke A, Treasure J. The use of guided self-help incorporating a mobile component in people with eating disorders: a pilot study. Eur Eat Disord Rev 2013;21:315-22.

38. Faul F, Erdfelder E, Lang AG, et al. G*Power 3: a flexible statistical power analysis program for the social, behavioral, and biomedical sciences. Behav Res Methods 2007;39:175-91.

39. Bundespsychotherapeutenkammer. BPtK-Studie zu wartezeiten in der abulanten psychotherapeutischen versorgung, 2011. http:// www.bptk.de/uploads/media/110622 BPtK-Studie Langfassung Wartezeiten-in-der-Psychotherapie_01.pdf (accessed 22 Aug 2016).

40. Hilbert A, Tuschen-Caffier B, Ohms M. Eating disorder examination: deutschsprachige version des strukturierten essstörungsinterviews. Diagnostica 2004;50:98-106.

41. Hilbert A, Buerger A, Hartmann AS, et al. Psychometric evaluation of the eating disorder examination adapted for children. Eur Eat Disord Rev 2013;21:330-9.

42. Graubner B. ICD-10-GM: Internationale statistische Klassifikation der Krankheiten und verwandter Gesundheitsprobleme. German modification. Köln: Dt. Ärzte-Verl, 2012.
43. Raevuori A, Hoek HW, Susser E, et al. Epidemiology of anorexia nervosa in men: a nationwide study of Finnish twins. PLoS One 2009;4:e4402.

44. Lavender JM, Brown TA, Murray SB. Men, muscles, and eating disorders: an overview of traditional and muscularity-oriented disordered eating. Curr Psychiatry Rep 2017;19:32.

45. Harris PA, Taylor R, Thielke R, et al. Research electronic data capture (REDCap)-a metadata-driven methodology and workflow process for providing translational research informatics support. J Biomed Inform 2009;42:377-81.

46. van Noort B, Karabasheva E, Porsch V, et al. Smartphone-App "Jourvie" als therapieunterstützungstool bei essstörungen: erste ergebnisse der benutzerfreundlichkeit und anwendbarkeit: poster. München: XXXIV DGKJP Kongress.

47. Herpertz S, Herpertz-Dahlmann B, Fichter M, et al. S3-Leitlinie diagnostik und behandlung der essstörungen. Berlin, Heidelberg: Springer Berlin Heidelberg, 2011.

48. Kv A, Bohus M. eds. Skills training for adolescents with emotion regulation problems - therapist manual]. Aufl ed. Stuttgart: Schattauer, 2015.

49. Bohus M, Wolf-Arehult M. Interaktives skillstraining für borderlinepatienten: das therapeutenmanual. aktualisierte und erwAufl. ed. Stuttgart: Schattauer, 2013

50. Kromeyer-Hauschild K, Wabitsch M, Kunze D, et al. Perzentile für den Body-mass-Index für das Kindes- und Jugendalter unter Heranziehung verschiedener deutscher Stichproben. Monatsschrift Kinderheilkunde 2001;149:807-18.

51. Hemmelmann C, Brose S, Vens M, et al. [Percentiles of body mass index of 18-80-year-old German adults based on data from the Second National Nutrition Survey]. Dtsch Med Wochenschr 2010;135:848-52.

52. Thiel A, Jacobi C, Horstmann S, et al. [A German version of the Eating Disorder Inventory EDI-2]. Psychother Psychosom Med Psychol 1997:47:365-76.

53. Salbach-Andrae H, Schneider N, Bürger A, et al. [Psychometric properties of the Eating Disorder Inventory (EDI-2) in adolescents]. Z Kinder Jugendpsychiatr Psychother 2010;38:219-28.

54. Beck AT, Steer RA, Brown GK. Manual for the beck depression inventory-II. . San Antonio: TX: Psychological Corporation, 1996:1. 82.

55. Kühner $\mathrm{C}$, Bürger $\mathrm{C}$, Keller $\mathrm{F}$, et al. [Reliability and validity of the Revised Beck Depression Inventory (BDI-II). Results from German samples]. Nervenarzt 2007;78:651-6.

56. Dolle K, Schulte-Körne G, O'Leary AM, et al. The Beck depression inventory-II in adolescent mental health patients: cut-off scores for detecting depression and rating severity. Psychiatry Res 2012;200:843-8.

57. Franke GH, Ankerhold A, Haase M, et al. Der Einsatz des Brief Symptom Inventory 18 (BSI-18) bei Psychotherapiepatienten. Psychother Psychosom Med Psychol 2011;61:82-6.

58. Franke GH. SCL-90-R. Die Symptom-Checkliste von Derogatis Deutsche Version. Göttingen: Beltz Test, 1995.

59. Laugwitz B, Held T, Schrepp M. Construction and Evaluation of a User Experience Questionnaire. Holzinger $\mathrm{A}$, ed. $\mathrm{HCl}$ and usability for education and Work. Berlin: Springer, 2008:63-76.

60. Kolar DR, Bürger A, Hammerle F, et al. Aversive tension of adolescents with anorexia nervosa in daily course: a case-controlled and smartphone-based ambulatory monitoring trial. BMJ Open 2014;4:e004703.

61. Watson D, Clark LA, Tellegen A. Development and validation of brief measures of positive and negative affect: the PANAS scales. J Pers Soc Psychol 1988;54:1063-70.

62. Krohne HW, Egloff B, Kohlmann CW, et al. Investigations with a German version of the positive and negative affect schedule (PANAS). Diagnostica 1996;42:139-56.

63. Zipfel S, Wild B, Groß G, et al. Focal psychodynamic therapy, cognitive behaviour therapy, and optimised treatment as usual in outpatients with anorexia nervosa (ANTOP study): randomised controlled trial. Lancet 2014;383:127-37.

64. Kolar DR, Hammerle F, Jenetzky E, et al. Bridging the gap: smartphone-based support between sessions for adolescent outpatients with anorexia nervosa - a randomised controlled trial protocol. European Eating Disorders Review 2016;24:E21.

65. Lloyd S, Yiend J, Schmidt U, et al. Perfectionism in anorexia nervosa: novel performance based evidence. PLoS One 2014;9:e111697. 